\title{
Cherry-picking the Wrong Patients has to be Avoided at all Cost!
}

\author{
Johanna M. Ospel11,2 • Mayank Goyal'1,3 iD \\ Received: 21 January 2020 / Accepted: 24 January 2020 / Published online: 7 February 2020 \\ (c) Springer-Verlag GmbH Germany, part of Springer Nature 2020
}

We recently wrote about the influence of age and the Alberta Stroke Program Early CT Score (ASPECTS) on endovascular treatment (EVT) decisions in acute stroke, and hypothesized that by excluding old patients in low ASPECTS EVT trials, the overall treatment effect size of EVT will decrease, thereby possibly leading to a false negative trial outcome. In their response letter, the TENSION investigators argued that there is a possibility that while the overall outcomes are worse in older patients, the treatment effect might actually be preserved [1]. We agree with the TENSION investigators that there is a possibility that the EVT treatment effect is not influenced by patient age; however, a possible effect modification cannot be completely disregarded, since only few patients with ASPECTS $<5$ were included in past trials, and our knowledge about EVT benefit in this subgroup is limited by a very small sample size. Post-stroke outcomes in old patients are compromised by several factors, such as physical and cognitive comorbidities and lack of social support networks [2], which could negatively affect treatment effect size. Most importantly, we have to ask ourselves the question: what is a good outcome? It is known that older patients will suffer from worse outcomes compared to young patients, with and without EVT [1]. Let us assume that the treatment effect is preserved in older patients: EVT would then in many cases lead to a change in the 90-day modified Rankin score (mRS) from 4 to 3 or from 5 to 4 . Although there clearly is a treatment effect, the question whether such a result is worth perform- ing EVT remains unclear and depends on multiple factors including individual patient preferences and availability of support networks. It is definitely a question that needs to be answered. Thus, we completely agree with the TENSION investigators that the single most important thing to do now is to convince physicians to enroll as many eligible patients as possible across all age groups, in order to obtain a solid and valid trial result.

\section{Compliance with ethical guidelines}

Conflict of interest J.M. Ospel is supported by the University of Basel Research Foundation, Julia Bangerter Rhyner Foundation and "Freiwillige Akademische Gesellschaft Basel" Foundation. M. Goyal is a consultant for Medtronic, Stryker, Microvention, GE Healthcare and Mentice.

Ethical standards For this article no studies with human participants or animals were performed by any of the authors. All studies performed were in accordance with the ethical standards indicated in each case.

\section{References}

1. Goyal M, Menon BK, van Zwam WH, Dippel DW, Mitchell PJ, Demchuk AM et al. Endovascular thrombectomy after large-vessel ischaemic stroke: a meta-analysis of individual patient data from five randomised trials. Lancet. 2016;387:1723-31.

2. Lui SK, Nguyen MH. Elderly stroke rehabilitation: overcoming the complications and its associated challenges. Curr Gerontol Geriatr Res. 2018. https://doi.org/10.1155/2018/9853837
Mayank Goyal

mgoyal@ucalgary.ca

1 Department of Clinical Neurosciences, University of Calgary, Calgary, Canada

2 Division of Neuroradiology, Clinic of Radiology and Nuclear Medicine, University Hospital Basel, University of Basel, Basel, Switzerland

3 Department of Diagnostic Imaging, University of Calgary, Calgary, Canada 